\title{
Effect of Regular Gum Chewing on Levels of Anxiety, Mood, and Fatigue in Healthy Young Adults
}

\author{
Akiyo Sasaki-Otomaru ${ }^{1}{ }_{*}$, Yumiko Sakuma $^{2}$, Yoshiko Mochizuki $^{3}$, Sadayo Ishida $^{4}$, \\ Yuka Kanoya $^{5}$ and Chifumi Sato ${ }^{2}$
}

\author{
${ }^{I}$ Department of Fundamental Nursing and Life Support, Division of Comprehensive Health Nursing Sciences, Graduate \\ School of Health Care Sciences, Tokyo Medical and Dental University, Tokyo, Japan \\ ${ }^{2}$ Department of Analytical Health Science, Graduate School of Health Care Sciences, Division of Comprehensive Health \\ Nursing Sciences, Tokyo Medical and Dental University, Tokyo, Japan \\ ${ }^{3}$ Tokai University Junior College of Nursing and Medical Technology, Hiratsuka-city, Japan \\ ${ }^{4}$ Faculty of Nursing, Yamanashi Prefectural University, Kofu-city, Japan \\ ${ }^{5}$ Course of Community Health Nursing, School of Nursing, Yamagata University Faculty of Medicine, Yamagata-city, \\ Japan
}

\begin{abstract}
Introduction: The effect of regular gum chewing on psychological status is unknown. The purpose of this study was to examine the effect of gum chewing for fourteen days on psychological status and physical and mental fatigue in healthy young adults.

Methods: We assigned 50 volunteers randomly to an intervention group $(\mathrm{n}=26)$ and a control group $(\mathrm{n}=24)$. Participants in the intervention group were requested to chew the gum twice per a day for fourteen days. The volunteers were required to complete a questionnaire related to lifestyle for baseline assessment. The State-Trait Anxiety Inventory (STAI), the Profile of Mood State (POMS), the World Health Organization Quality of Life 26, and assessment of physical and mental fatigue by visual analog scale were used at baseline, 2 weeks (after intervention), and 4 weeks (follow-up).

Results: At 2 weeks, the score of state anxiety was significantly lower in the intervention group than the control group. The intervention participants' scores of depression-dejection, fatigue and confusion in POMS were better than the control group scores. Mental fatigue were also relieved after the intervention. At 4 weeks, there were no significant differences between both groups.

Conclusion: Fourteen days' gum chewing may improve the levels of anxiety, mood and fatigue.
\end{abstract}

Keywords: Gum, chewing, anxiety, mood, fatigue.

\section{INTRODUCTION}

Mastication involves rhythmic and voluntary movements of lower jaw by the masticatory muscles bringing the process by which food is crushed and ground by teeth. This process is the first step of digestion and increases salivary flow. Tooth loss and asthenia of masticatory muscles causes impairment of masticatory function and chewing disability.

Animal experiments have shown that tooth loss or longterm soft-diet feeding causes a decrease in learning and memory ability [1-3]. A previous study also revealed that soft-diet feeding after a weaning period reduces synaptic formation in the cerebral cortex and impairs the ability of spatial learning in adulthood [4]. Biting during restraint ex-

*Address correspondence to this author at the Department of Fundamental Nursing and Life Support, Division of Comprehensive Health Nursing Sciences, Graduate School of Health Care Sciences, Tokyo Medical and Dental University 1-5-45, Yushima, Bunkyo, Tokyo, 113-8510 Japan;

Tel/Fax: +81-3-5803-5344; E-mail; sakiahs@ @md.ac.jp posure suppresses stress-induced catecholamine concentrations in the blood [5]; numbers of neuronal nitric oxide synthase-positive neurons, which respond to stressors, are reduced in the hypothalamus [6]. These studies imply that masticatory activity induced a positive anti-stress effect in animals.

Mastication is regulated by a neural population in the brainstem and a neural network including several brain regions. Gum chewing has been shown to be associated with increased cerebral blood flow in humans [7-9], and several studies have reported increased cerebral activity following gum chewing [10-12]. Thus, the beneficial effects of chewing on cerebral activity have been suggested, even though the mechanism of these effects is controversial.

In epidemiological studies, oral function and status have been shown to be related to physical, mental, and social health. In particular, chewing ability has been shown to influence activities of daily living [13], cognitive status [14], and quality of life in elderly people [15]. However, these 
results have been reported in cross-sectional studies. Prospective studies are required to determine the association between chewing ability and health status.

We have previously reported that the mental endurance on the psychological stress test of elementary school students improved after eating hard gumdrops for 28 days [16]. Although the gumdrop were harder than a general gum on the market in this study, it were not able to continue chewing like a gum. In a recent report, the perceived level of stress was found to decrease after a gum-chewing period and increase after gum-abstain period [17].

Thus, gum chewing is a popular and convenient way to help relieve stress and improve concentration. Regular gum chewing may influence mental health, although the effects of gum chewing on mood or fatigue were unclear. Promoting mental health is a global public health issue. However, few studies have investigated the relationship between chewing and mental health status. The purpose of this study was to examine the effect of gum chewing for 14 days on psychological status and fatigue in healthy young adults.

\section{METHODS}

\section{Participants}

The present study was conducted between June and September 2009 at two nursing colleges in Japan. Participants were recruited from April 2009 by putting up a poster on the bulletin board at the two schools. Fifty ( 3 males, 47 females) healthy adults participated in this study. Inclusion criteria included no oral diseases such as caries or TMJ arthrosis, the ability to chew a piece of gum for at least $5 \mathrm{~min}$ at a time and being less than 40 years old. Exclusion criteria included not being able to chew gum for any reason and milk allergy; the latter exclusion criterion was included because of the gum ingredients. No volunteers were excluded on the basis of these criteria. The participants were then randomly allocated into the intervention and control groups.

\section{Procedures}

This study was approved by the ethics committee of the Faculty of Medicine, Tokyo Medical and Dental University.

We assigned the participants randomly to the intervention group $(n=26)$ and the control group $(n=24)$ according to a list of random computer-generated numbers. Selfadministered questionnaires were used in the present study. The participants were asked age, sex, hours of sleep, the frequency of gum chewing, whether the person studied while gum chewing, and whether he or she took a break while chewing. We also asked the participants with the Visual Analog Scale (VAS) that they chew diet well as statement of "subjective dietary chewing". In addition, the participants were asked to complete the State-Trait Anxiety Inventory (STAI), the short-form Profile of Mood States (POMS), the World Health Organization QOL 26 (WHO-QOL26), and an assessment of fatigue with the Visual Analog Scale (VAS). We repeated the assessment of STAI (state -anxiety only), POMS, WHOQOL26, and fatigue at 2 weeks (after intervention) and 4 weeks (follow-up). Participants were asked to mail the questionnaires with a postal envelope at each time point. No further intervention was offered except the 14 days' gum chewing.

\section{Intervention}

The participants of the intervention group were asked to chew gum twice per day for 14 days. Recaldent Gum with mint flavor (Cadbury Japan, Tokyo, Japan) was used. We encouraged the participants to chew the gum for at least 5 min at a time. We also explained that they could chew the gum at any time and at their own pace. The frequency and length of time of gum chewing were based on previous studies and the consumption recommended by the gum manufacturer [16, 18-20].

The ingredients of Recaldent Gum with mint flavor were as follows: maltitol, syrup, calcium casein peptone-calcium phosphate, gum base, xylitol, aspartame, L-phenylalanine, acesulfame potassium, natural and artificial flavoring, gelatin, gum arabic, mannitol, candelilla wax, and soy lecithin. The weight of a single piece is $1.36-1.37 \mathrm{~g}$. To ensure compliance with the gum chewing intervention, participants in the intervention group were asked to record information about the gum chewing. We assessed how many days and how many times they chewed. Adherence was calculated by dividing the total number of times gum was chewed by 14 (days) and multiplying this value by 2 (times per a day).

The control participants were provided with a mint product (MINTIA; ASAHI FOOD \& HEALTHCARE CO., Tokyo, Japan) to prevent them from dropping out. To avoid the influence of chewing and flavor [7,21], we selected a product with a mint taste that resembled the taste of the gum. The ingredients of the mint product were as follows: lemon juice powder, parsley seed oil, sorbitol, aspartame, Lphenylalanine, acesulfame potassium, flavoring, silicon dioxide fine powder, sucrose ester, gardenia yellow, green tea extract. The weight of a single piece is $0.14 \mathrm{~g}$. We asked the participants in the control group to have a mint whenever they wanted and not to refrain gum chewing. We checked whether they chewed gum more than once a day during the intervention period (baseline through $2 \mathrm{w}$ ) and the follow-up period ( $2 \mathrm{w}$ through $4 \mathrm{w})$.

\section{Outcome Measurements}

\section{Anxiety}

Anxiety was assessed using Spielberger's STAI. It consists of two parts, state anxiety and trait anxiety [22, 23]. The state anxiety scale consists of 20 statements that evaluate how the respondent feels "right now, at this moment." The trait anxiety scale consists of 20 statements to evaluate how the respondent feels "generally." The items were developed for responses on a 4-point, Likert-type scale. The trait anxiety score was used only as a baseline assessment in this study.

\section{Mood}

The POMS is a self-administered adjective checklist measuring transient mood states [24]. We used the shortform POMS Japanese edition, which includes 30 items and a coded 5-point scale [25]. The POMS has the six measurement factors for "tension-anxiety," "depression-dejection," "anger-hostility," "vigor," "fatigue," and "confusion." 


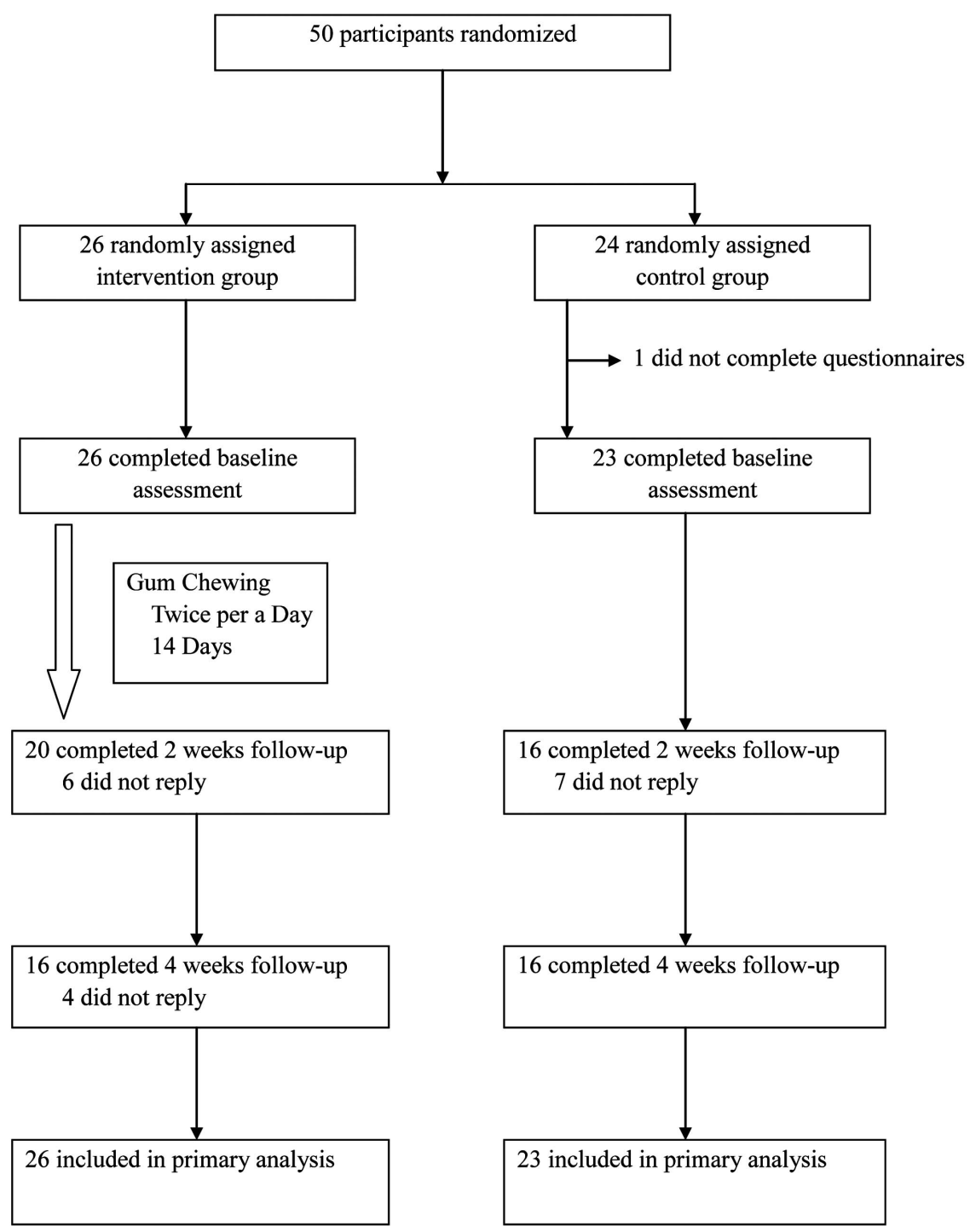

Fig. (1). Flow of Participants through the Study.

\section{Quality of Life}

The QOL was assessed using the Japanese version of the WHO-QOL26 [26]. The WHOQOL26, a generic healthrelated QOL evaluation, measures overall QOL and four separate domains of health: physical, psychological, social relationships, and environment [27]. Each question is graded on scale of 1-5 points. The lower the score is, the greater the disability.

\section{Assessment of Fatigue}

Physical and mental fatigue were evaluated subjectively by using the VAS. The VAS consists of a 100-mm line, anchored with the extremes of fatigue strength as "no fatigue" and "worst fatigue possible". Participants were asked to rate how they felt at that precise moment.

\section{Statistical Analysis}

All analyses were performed using SPSS 17.0 for Windows (SPSS Japan Inc, Tokyo, Japan). We used the $t$ test, the chi-square test, and the Mann-Whitney U test to compare the baseline characteristics of intervention and control participants. These testing were two-tailed, at the 0.05 significance

level. The two follow-up time points were conducted at 2 and 4 weeks. The primary analysis was based on intentionto-treat analysis. The student $t$ test with Bonferroni correction $(p<0.016)$ was used to detect the differences between the intervention group and the control group at baseline, 2 weeks, and 4 weeks.

\section{RESULTS}

The Fig. (1) shows the flow of participants through the study. Fifty participants were enrolled in the study, including 26 in the intervention group and 24 in the control group. A woman dropped out because she did not complete the questionnaires at baseline. At 2 weeks, 6 intervention and 7 control participants did not reply. At 4 weeks, 13 drop-outs of 2 weeks and another 3 intervention participants did not reply. Forty-nine participants were included in the analysis, and thirty-three participants completed the study. For the 16 drop-outs, the scores were higher for two factors of the POMS than they were for the 33 participants (depressiondejection 7.7 for the drop-outs $v s 4.8$ for the remaining participants, $\mathrm{p}=0.036$; confusion 10.7 vs 8.0 , respectively; $\mathrm{p}=$ $0.018)$. However, the drop-outs did not differ with regard to other characteristics and measurements at baseline. 
Table 1. Characteristics of Participants at Baseline

\begin{tabular}{|c|c|c|}
\hline & Intervention & Control \\
\hline Characteristics & $(n=26)$ & $(\mathbf{n}=\mathbf{2 3})$ \\
\hline Age, mean (SD), y & $21.5(5.0)$ & $20.1(2.7)$ \\
\hline \multicolumn{3}{|l|}{ Institution (school) } \\
\hline A & $14(54 \%)$ & $10(43 \%)$ \\
\hline usually & $5(19 \%)$ & $3(13 \%)$ \\
\hline often & $13(50 \%)$ & $12(52 \%)$ \\
\hline rarely & $7(27 \%)$ & $7(30 \%)$ \\
\hline not at all & $1(4 \%)$ & $1(4 \%)$ \\
\hline not at all & $6(23 \%)$ & $3(13 \%)$ \\
\hline \multicolumn{3}{|l|}{ Take a break while gum chewing } \\
\hline usually & $2(7 \%)$ & $5(22 \%)$ \\
\hline often & $9(35 \%)$ & $7(30 \%)$ \\
\hline rarely & $9(35 \%)$ & $6(26 \%)$ \\
\hline not at all & $6(23 \%)$ & $5(22 \%)$ \\
\hline \multicolumn{3}{|c|}{ Subjective dietary chewing (VAS), mean (SD) } \\
\hline baseline & $44.9(20.1)$ & $42.0(24.5)$ \\
\hline $2 \mathrm{w}$ & $\mathrm{n}=2070.5(22.8)$ & $\mathrm{n}=1638.8(19.0)$ \\
\hline
\end{tabular}

\section{Characteristics of the Participants}

Table 1 shows the participants' characteristics and life style. Forty-nine participants completed the baseline assessment. The mean age was 20.9 years $(S D=4.1)$, and only three were men. More than half of the participants were regular gum chewers before participating in the study. There was no significant difference in trait-anxiety of STAI between the intervention and control groups.

\section{Differences in the Scores for Anxiety, Mood, and Fatigue in Two Groups}

All measurements at baseline were similar in both groups (Table 2). After 2 weeks, outcome data were obtained for 36 (72\%) participants. All of the 36 participants were found to have chewed gum for more than 13 days. Adherence was less than $80 \%$ in only 2 participants. In the control group, two subjects had chewed a gum more than once a day during the intervention period.

At 2 weeks, the score of state anxiety was significantly lower in the intervention group than in the control group $(\mathrm{p}<$ 0.016). The scores for depression-dejection, fatigue, and confusion for POMS were significantly lower in the intervention group than in the control group $(\mathrm{p}<0.016)$. Mental fatigue scores for the intervention participants were significantly different from those of the control participants (Table 2).

In regard to the QOL scores, there were no significant differences except for physical domain between the two groups.

At the follow-up assessment (4 weeks), 32 participants replied to the questionnaires. There were no significant differences in all outcomes between the intervention and control groups (Table 2).

Eight subjects in the intervention group had chewed a gum more than once a day during the follow-up period. At 4 weeks, the mental states of gum chewers $(n=8)$ were not different significantly than those of no gum chewers $(n=24)$.

At 2 and 4 weeks, the states of subjective dietary chewing in the intervention group differed significantly from those in the control group. The participants in the intervention group think that they chewed diet well.

\section{DISCUSSION}

The aim of this study was to examine the effects of gum chewing for 14 days on mental health parameters. Stateanxiety, mood, and fatigue were found to be improved after 
Table 2. Comparisons of Anxiety, Mood and Fatigue between Intervention and Control Groups

\begin{tabular}{|c|c|c|c|c|c|c|c|c|}
\hline & & \multicolumn{3}{|c|}{ Intervention } & \multicolumn{3}{|c|}{ Control } & $\mathbf{p}$ \\
\hline \multicolumn{9}{|l|}{ [STAI] } \\
\hline \multirow[t]{2}{*}{ State anxiety } & baseline & 25 & 44.4 & 7.6 & 23 & 44.1 & 9.0 & \\
\hline & $2 \mathrm{w}$ & 20 & 38.9 & 7.5 & 16 & 49.6 & 11.3 & $*$ \\
\hline \multirow[t]{3}{*}{ Tension-Anxiety } & baseline & 26 & 9.0 & 5.3 & 23 & 9.9 & 4.9 & \\
\hline & $2 w$ & 20 & 5.3 & 3.3 & 16 & 8.3 & 4.8 & \\
\hline & $4 w$ & 16 & 6.1 & 4.4 & 16 & 7.3 & 5.3 & \\
\hline Depression-Dejection & baseline & 26 & 6.2 & 4.4 & 23 & 5.4 & 4.6 & \\
\hline \multirow[t]{3}{*}{ Anger-Hostility } & baseline & 26 & 4.7 & 4.3 & 23 & 5.1 & 4.6 & \\
\hline & $2 w$ & 20 & 3.5 & 2.9 & 16 & 5.9 & 4.3 & \\
\hline & $4 w$ & 16 & 3.9 & 3.0 & 16 & 4.7 & 4.6 & \\
\hline \multirow[t]{3}{*}{ Vigor } & baseline & 26 & 8.0 & 3.6 & 23 & 7.3 & 4.1 & \\
\hline & $2 w$ & 20 & 8.7 & 3.3 & 16 & 6.8 & 3.3 & \\
\hline & $4 w$ & 16 & 9.1 & 3.7 & 16 & 7.6 & 4.7 & \\
\hline \multirow[t]{3}{*}{ Fatigue } & baseline & 26 & 10.6 & 4.7 & 23 & 12.0 & 5.4 & \\
\hline & $2 \mathrm{w}$ & 20 & 6.8 & 3.6 & 16 & 10.3 & 4.3 & $*$ \\
\hline & $4 w$ & 16 & 8.8 & 4.9 & 16 & 9.3 & 4.7 & \\
\hline [Physical fatigue] & $4 w$ & 16 & 49.9 & 30.1 & 16 & 49.1 & 26.5 & \\
\hline \multirow[t]{3}{*}{ [Mental fatigue] } & baseline & 26 & 60.9 & 22.6 & 23 & 65.7 & 20.8 & \\
\hline & $2 w$ & 20 & 34.3 & 22.4 & 16 & 60.6 & 21.8 & $*$ \\
\hline & $4 w$ & 16 & 41.4 & 29.7 & 16 & 59.6 & 20.4 & \\
\hline \multicolumn{2}{|c|}{ t-test with Bonferroni correction } & & & & & & & $\mathrm{p}<0.016$ \\
\hline
\end{tabular}

gum chewing in the intervention group as compared with the control group. At the follow-up assessment, the scores for the intervention and control groups were similar. These results suggest that the improvement by the intervention may not be maintained afterwards.

Our results support the hypothesis that regular gum chewing has an effect on psychological status. This trial examined the effect of gum chewing on both experimental stress and also social-related stress. The results of the previous studies that examined the effects of gum chewing on psychological stress test were controversial. Scholey et al. [28] investigated the effects of gum chewing on task-induced stress. In this study, gum chewing was found to be associated with decreased levels of self-rated anxiety and stress and a reduction in salivary cortisol concentrations. Based on the Scholey study, the Toney's participants performed an insoluble anagram task followed by a soluble anagram task [29]. The performance of these tasks and the levels of stress were found to be similar with or without gum chewing.

In the present study, the scores for state -anxiety in the intervention group were better than those in the control group after gum chewing for 14 days. The scores for depression-dejection, fatigue, confusion in POMS and fatigue on VAS were also significantly different in both groups. On the other hand, the scores of vigor in POMS and the QOL scores except physical domain were similar between the two groups. These results suggest that regular gum chewing improves negative feelings rather than promotes positive feel- 
ings. The WHOQOL26 instrument evaluates not only physical and mental satisfactions but also the social and environmental aspects. The WHO states that this instrument assesses the individual's perceptions in the context of their culture and value systems, as well as their personal goals, standards, and concerns [26]. A longitudinal intervention is needed to confirm the influence on QOL.

The participants in the present study were nursing students. Generally, nursing students have high levels of stress and anxiety in the clinical learning environment [30-32]. The present study was carried out while the students were not involved in clinical training. Their levels of anxiety and mood were similar to those of the previous Japanese studies $[25,30]$. Even though many research articles have been published on a variety of strategies to help decrease students' high levels of anxiety in the clinical learning environment [33], few researchers have been successful in finding ways of decreasing student anxiety. Therefore, our results indicating that daily gum chewing influenced the anxiety levels and mood of nursing students seem to be important.

Gum chewing also was shown to relieve mental fatigue in the present study. Fatigue is related to stress [34], and chronic fatigue has been found to induce serious conditions [35]. Lasting fatigue is difficult to recover from by simple rest or task moderation. Our previous study showed that gum chewing during the time of a psychological stress test improved physical and mental fatigue after the test [36]. According to the Japanese National Survey of Health in 2004 [37], 49\% of individuals aged 12 years or older reported experiencing stress in their daily lives. Finding ways to recover from fatigue after a stressful event may be helpful in stress management.

In Zibell's study[17], different intervention periods were applied for regular chewer and seldom chewer. The constant gum chewing was conducted in our study regardless of chewing habit, and then this gum chewing may affect the participant's mental health. In this study, the quantitative frequency of gum chewing before the study were not clarify because the 4-points Likert scale to examine how the participants were familiar with gum chewing. Further investigation is necessary to better understand how cessation of chewing affects mental health of regular chewers and whether the improvement of mental state was larger in seldom chewers than regular chewers.

Various mental health programs have been developed to try to improve psychological status. Hollingworth (1939) described chewing as a technique of relaxation [38]. Yoga also has been reported to improve levels of anxiety [39, 40]. Aroma has been shown to influence mood [21], and meditation has been shown to reduce stress and improve mood [41]. To get the beneficial effects of these techniques, participants must adhere to the technique and motivated. As gum chewing does not require any special preparation, skills, or equipments, it may be widely accepted.

The present study has some limitations. The sample size was small, and there was a selection bias in participants being recruited from only two colleges. We did not power analysis because there were no available preliminary studies that were referred to sample size, we recruited volunteers as much as possible. Also, the participants had motivation and interest in the study and gum chewing. Therefore, the results may not be applicable to general population who has no incentive.

To prevent dropout of the subjects, the intervention term was designed to use the minimum number of days that would allow us to estimate an effect. We did not instruct the control participants not to bite a mint product. Even if they bite mint product, however, this mint would dissolve in their mouth after a few bite. Therefore, chewing effect in this group seemed to be minimal. Also, we did not ask them to refrain gum chewing during the intervention period. Although two control participants chewed a gum more than once a day, we were not able to analyze because of small sample size.

Since there were no available data how the duration affects mental status, we adopted " 5 minute" chewing. Consequently, the total duration of chewing may variety. In addition, the scores of subjective dietary chewing were different significantly between the intervention and control groups at 2 weeks and 4 weeks assessment. The intervention participants would come to chew in daily life by allocating the intervention group. Further studies needed to clarify not only the effects of duration and intervention period of gum chewing, but also dietary habit.

The subjects in this study were healthy condition in some psychological outcomes. To apply mental health care or stress-management, the effect of gum chewing will be examined in people under stressful environment. Another limitation of this study was that a double-blind design could not be applied because of the nature of this kind of interventions.

In conclusion, the results of the present study show that 14 days gum chewing improves the levels of anxiety, mood, and fatigue in healthy young adults.

\section{ACKNOWLEDGEMENTS}

We are grateful to Prof. Takanori Kitamura at Tokai University for his generous support to recruit the participants.

This study was supported by the Grant-in-Aid for Scientific Research (C), No. 20500595 (KAKENHI) from the Ministry of Education, Culture, Sports, Science and Technology in Japan.

\section{REFERENCES}

[1] Kawamura S. The effect of food consistency on conditioned avoidance response in mice and rats. Shika Kiso Igakkai Zasshi (Jpn J Oral Biol) 1989; 31(1): 72-82. [in Japanese]

[2] Umeda K, Sawaki Y, Ito M, et al. Effects of teeth loss on learning and memory ability in adult rats. KoKubyo Gakkai Zasshi (J Jpn Stomatol Soc) 1991; 40(2): 377-83. [in Japanese]

[3] Tsutsui K, Kaku M, Motokawa M, et al. Influences of reduced masticatory sensory input from soft-diet feeding upon spatial memory/learning ability in mice. Biomed Res 2007; $28: 1-7$.

[4] Yamamoto T, Hirayama A. Effects of soft-diet feeding on synaptic density in the hippocampus and parietal cortex of senescenceaccelerated mice. Brain Res 2001; 902: 255-63.

[5] Hori N, Yuyama N, Sasaguri K, et al. Effects of biting and response of sympathetic nerve system during the restraint stress. Bull Kanagawa Dent Coll 2004; 32(2): 123-5.

[6] Ishii H, Hori N, Saruta J, Sasaguri K, Lee MC, Sato S. Effects of biting on nNOS in the paraventricular nucleus during the restraint stress. Bull Kanagawa Dent Coll 2004; 32(2): 133-5. 
[7] Momose I, Nishikawa J, Watanabe T, et al. Effect on electroencephalogram of chewing flavored gum. Psychiatry Clin Neurosci 2000; 54: 645-51.

[8] Sesay M, Tanaka A, Ueno Y, Lecaroz P, De Beaufort DJ. Assessment of regional cerebral blood flow by xenon-enhanced computed tomography during mastication in human. Keio J Med 2000; 49(1): A125-8.

[9] Onozuka M, Fujita M, Watanabe K, et al. Mapping brain region activity during chewing: a functional magnetic resonance imaging study. J Dent Res 2002; 81: 743-6.

[10] Fang M, Li JC, Lu G, Gong XY, Yew DT. A fMRI study of agerelated differential cortical patterns during cued motor movements. Brain Topogr 2005; 17: 127-37.

[11] Hirano Y, Obata T, Kashikura K, et al. Effects of chewing in working memory processing. Neurosci Lett 2008; 436: 189-92.

[12] Sakamoto K, Nakata H, Kakigi R. The effect of mastication on human cognitive processing: a study using event-related potentials. Clin Neurophysiol 2009; 120: 41-50.

[13] Miura H, Kariyasu M, Yamasaki K, Arai Y, Sumi Y. Relationship between general health status and the change in chewing ability: a longitudinal study of the frail elderly in Japan over a 3-year period. Gerodontology 2005; 22: 200-5.

[14] Miura H, Yamasaki K, Kariyasu M, Miura K, Sumi Y. Relationship between cognitive function and mastication in elderly females. J Oral Rehabil 2003; 30: 808-11.

[15] Miura H, Miura K, Mizugai H, Arai Y, Umenai T, Isogai E. Chewing ability and quality of life among the elderly residing in a rural community in Japan. J Oral Rehabil 2000; 27:731-4.

[16] Otomaru A, Kanoya Y, Watanabe $\mathrm{H}$, et al. The effect of original gumdrop chewing on psychological status and oral function of children in an elementary school. Nippon Sosyaku Gakkai Zasshi (J Jpn Soc Masticatory Sci Health Promotion) 2003; 13(2): 67-76. [in Japanese].

[17] Zibell S. Madansky E. Impact of gum chewing on stress levels: online self-perception research study. Curr Med Res Opin 2009; 25: 1491-500.

[18] Kiliaridis S, Tzakis MG, Carlsson GE. Effects of fatigue and chewing training on maximal bite force and endurance. Am J Orthod Dentofacial Orthop 1995; 107: 372-8.

[19] Watanabe H, Arakawa S, Tominaga Y, et al. The effect of a novel masticatory foodstuffs on human oral and immunological functions. Nippon Sosyaku Gakkai Zasshi (J Jpn Soc Masticatory Sci Health Promotion) 2001; 10(2): 101-5. [in Japanese].

[20] Watanabe H, Kanoya Y, Kusaka K, et al. Novel chewable foodstuffs for the elderly: its effect on oral function and memory acquirement. Nippon Sosyaku Gakkai Zasshi (J Jpn Soc Masticatory Sci Health Promotion) 2003; 3(1): 37-43. [in Japanese].

[21] Moss M, Hewitt S, Moss L, Wesnes K. Modulation of cognitive performance and mood by aromas of peppermint and ylang-ylang. Int J Neurosci 2008; 118: 59-77.

[22] Spielberger CD. State-Trait Anxiety Inventory (STAI). Redwood City: Mind Garden 1983.
[23] Hidano T, Fukuhara M, Iwasaki S, Soga S, Spielberger CD. The STAI manual. Tokyo: Jitsumu-Kyoiku-Syuppan 2000.

[24] McNair DM, Lorr M, Droppleman LF. Profile of Mood States Manual. San Diego: Educational and Industrial Testing Service 1971.

[25] Yokoyama K. Guide to a short version of the POMS and representative case descriptions. Tokyo: Kaneko Publishing, Inc 2005.

[26] Tazaki M, Nakane Y. The manual of WHO quality of life 26. Tokyo: Kaneko Publishing Inc 1997.

[27] WHOQOL Group. Development of the World Health Organization WHOQOL-BREF quality of life assessment. Psychol Med 1998 28: 551-8.

[28] Scholey A, Haskell C, Robertson B, Kennedy D, Milne A, Wetherell M. Chewing alleviates negative mood and reduces cortisol during acute laboratory psychological stress. Physiol Behav 2009; 97: 304-12.

[29] Torney KL, Johnson JA, Miles C. Chewing gum and impasseinduced self-reported stress. Appetite 2009; 53: 414-7.

[30] Mizuguchi K, Shimonaka J, Nakasato K. Japanese version of the STAI manual. Kyoto: Sankyobo 1991.

[31] Elliott M. The clinical environment: A source of stress for undergraduate nurses. Aust J Adv Nurs 2002; 20: 34-8.

[32] Cook LJ. Inviting teaching behaviors of clinical faculty and nursing students' anxiety. J Nurs Educ 2005; 44: 156-61.

[33] Moscaritolo ML. Interventional strategies to decrease nursing student anxiety in the clinical learning environment. J Nurs Educ 2009; 48: 17-23.

[34] Otsuka Y, Sasaki T, Iwasaki K, Mori I. Working hours, coping skill, and psychological health in Japanese daytime workers. Ind Health 2009; 47: 22-32.

[35] Fukuda K, Hickie I, Sharpe MC, Dobbins JG, Komaroff A, the International CHS Study Group. The chronic fatigue syndrome: a comprehensive approach to its definition and study. Ann Intern Med 1994; 121: 953-9.

[36] Sasaki-Otomaru A, Sakuma Y, Kanoya Y, Sato C. The effects of gum chewing on work efficiency and fatigue; a preliminary study. Nippon Kenko Igakkai Zasshi (J Japan Health Med Assoc) 2009; 18(1): 24-30. [in Japanese].

[37] Japanese Ministry of Health and Welfare: National Survey of Health 2004. Tokyo: Kosei Toukei Kyoukai, 2006.

[38] Hollingworth HL. Chewing as a technique of relaxation. Science 1939; 90: 385-7.

[39] Javnbakht M, Hejazi KR, Ghasemi M. Effects of yoga on depression and anxiety of women. Complement Ther Clin Pract 2009; 15: $102-4$.

[40] Subramanya P, Telles S. Effect of two yoga-based relaxation techniques on memory scores and state anxiety. Biopsychosoc Med 2009; $3: 8$.

[41] Speca M, Carlson LE, Goodey E, Angen M. A randomized, waitlist controlled clinical trial: the effect of a mindfulness meditationbased stress reduction program on mood and symptoms of stress in cancer outpatients. Psychosom Med 2000; 62: 613-22.

\begin{tabular}{lrl}
\hline Received: February 1, 2011 & Revised: June 17, 2011 & Accepted: June 20, 2011 \\
(C) Sasaki-Otomaru et al.; Licensee Bentham Open. &
\end{tabular}

(C) Sasaki-Otomaru et al.; Licensee Bentham Open .

This is an open access article licensed under the terms of the Creative Commons Attribution Non-Commercial License (http://creativecommons.org/licenses/by-nc/3.0/) which permits unrestricted, non-commercial use, distribution and reproduction in any medium, provided the work is properly cited. 